\title{
Attenuation of spinal cord ischemia and reperfusion injury by erythropoietin
}

\author{
Phillip D. Smith, MD, ${ }^{a}$ Ferenc Puskas, MD, PhD, ${ }^{\mathrm{b}}$ David A. Fullerton, MD, ${ }^{\mathrm{a}}$ Xianzhong Meng, MD, PhD, ${ }^{\mathrm{a}}$ \\ Doug Cho, BS, ${ }^{\mathrm{a}}$ Joseph C. Cleveland, Jr, MD, ${ }^{\mathrm{a}}$ Michael J. Weyant, MD, ${ }^{\mathrm{a}}$ and T. Brett Reece, MD ${ }^{\mathrm{a}}$
}

\begin{abstract}
Background: Paraplegia remains a devastating complication for patients undergoing thoracic aortic procedures. Although surgical adjuncts have evolved to reduce the risk of paraplegia, no pharmacologic therapies have proven efficacious in attenuating spinal cord ischemia-reperfusion injury. Effects of erythropoietin in spinal cord ischemia-reperfusion injury, however, have not yet been elucidated. We hypothesized that pretreatment with erythropoietin would attenuate functional and cytoarchitectural spinal cord injury related to high-risk aortic procedures.
\end{abstract}

\begin{abstract}
Methods: Adult male mice were subjected to ischemia-reperfusion. Aortic arch and proximal left subclavian arteries were clamped for 5 minutes; animals were observed for 48 hours. Neurologic scores of hind limb function were assessed every 12 hours. Experimental groups consisted of treatment with erythropoietin 4 hours before crossclamping $(\mathrm{n}=7)$, ischemic controls $(\mathrm{n}=7)$, and sham ischemia (operation without crossclamping, $\mathrm{n}=6$ ). Thoracolumbar sections of spinal cord were removed after 48 hours and preserved for cytoarchitectural analysis.
\end{abstract}

Results: Mice pretreated with erythropoietin exhibited significant preservation of hind limb motor function. All mice without pretreatment were paralyzed at 48 hours. Mice with erythropoietin pretreatment had improved motor function; 3 had no measurable neurologic deficit at 48 hours. Histologic analysis in mice treated with erythropoietin showed markedly reduced neuronal cell injury.

Conclusions: Erythropoeitin preserves both function and histologic appearance in mice undergoing spinal cord ischemia-reperfusion. With further elucidation of mechanisms of protection and optimal administration, erythropoietin could become an important adjunct in reducing the incidence and severity of spinal cord injury related to aortic interventions. (J Thorac Cardiovasc Surg 2011;141:256-60)

Spinal cord ischemia-reperfusion injury remains a significant complication of thoracic aortic surgery. Lumbar drains, intercostal artery reimplantation, left heart bypass, and hypothermic circulatory arrest have all decreased rates of paraplegia and paraparesis after aortic surgery. No pharmacologic adjuncts, however, have proven efficacious in attenuating the metabolic or inflammatory insult to the spinal cord resulting from this ischemia-reperfusion injury.

Erythropoeitin, a $34-\mathrm{kDa}$ endogenous glycoprotein, is most widely recognized for its role in hematopoiesis. Erythropoietin is known to be upregulated in many tissues in times of hypoxic stress as a result of stabilization of hypoxia-inducible factor $1 \alpha$ (HIF $1 \alpha)$, which upregulates

From the Departments of Cardiothoracic Surgery ${ }^{\mathrm{a}}$ and Anesthesia, ${ }^{\mathrm{b}}$ University of Colorado, Denver, Colo.

Disclosures: Authors have nothing to disclose with regard to commercial support.

Read at the 36th Annual Meeting of The Western Thoracic Surgical Association, Ojai, Calif, June 23-26, 2010.

Received for publication June 18, 2010; revisions received Aug 27, 2010; accepted for publication Sept 17, 2010.

Address for reprints: Phillip D. Smith, MD, University of Colorado, Cardiothoracic Surgery, Mail Stop C310, 12631 E 17th Ave, Room 6601, Aurora, CO 80045 (E-mail: phillip.smith@ucdenver.edu).

0022-5223/\$36.00

Copyright (c) 2011 by The American Association for Thoracic Surgery doi:10.1016/j.jtcvs.2010.09.017 erythropoeitin expression. ${ }^{1}$ This upregulation of erythropoeitin causes predictable effects in hematopoietic tissues but appears to have cytoprotective effects independent of this activity. ${ }^{2}$ In the brain and spinal cord, this beneficial effect has been seen in animal models in which erythropoietin provides neuroprotection against many disease states, including cerebral ischemia-reperfusion. ${ }^{3}$ Specifically in animal stroke models, meta-analysis reveals a neuroprotective effect of erythropoeitin with smaller infarct sizes and improved functional outcomes after treatment. ${ }^{4}$

The mechanism of this neuroprotective effect has not been well elucidated in either the brain or the spinal cord. It is known that this effect is independent of the obvious hematopoietic effects of erythropoeitin ${ }^{5}$ and that erythropoeitin is beneficial in other solid organ systems after ischemia-reperfusion injury. This mechanism may involve erythropoeitin's positive feedback on HIF $1 \alpha$ expression, because this protein is increased after renal ischemiareperfusion with erythropoietin pretreatment. ${ }^{6}$ Upregulation of HIF $1 \alpha$ has been implicated in neuroprotection after mild to moderate cerebral ischemia ${ }^{7}$ but has never been directly studied in the spinal cord.

From these data, we hypothesized that erythropoeitin would have a neuroprotective effect on both functional 


\section{Abbreviations and Acronyms}

HIF $1 \alpha=$ hypoxia-inducible factor $1 \alpha$

Tris $=$ tris(hydroxymethyl)aminomethane

outcomes and histologic appearance after ischemia-reperfusion of the spinal cord, an effect that might be due to modulation of HIF1 $\alpha$ expression. The purposes of this study were to evaluate the efficacy of erythropoietin in neuroprotection of the spinal cord after ischemia-reperfusion in a murine model and to attempt to elucidate a neuroprotective mechanism.

\section{MATERIALS AND METHODS}

\section{Animals and Surgical Procedures}

The Animal Care and Use Committee at the University of Colorado at Denver Health Sciences Center approved all experiments, and this investigation conformed to the Guide for the Care and Use of Laboratory Animals published by the National Institutes of Health (www.nap.edu/catalog/5140. html). C57bl/6 male mice were obtained from Jackson Laboratory (The Jackson Laboratory, Bar Harbor, Me). Mice between 12 and 20 weeks old were used for experimentation. With $2 \%$ isoflurane anesthesia, a cervicothoracic approach was used to expose the aortic arch, as previously described. ${ }^{8,9}$ The aortic arch was crossclamped between the left common carotid artery and the left subclavian artery under direct visualization. Ischemia continued for 5 minutes, after which clamps were removed. An additional clamp was placed on the left subclavian artery. Disruption of distal aortic flow was confirmed with a laser Doppler blood flow monitor (Moor Instruments Ltd, Axminster, UK) to achieve a greater than $90 \%$ decrease in distal aortic flow measured at the femoral artery. An intraperitoneal dose of $400 \mathrm{IU} / \mathrm{kg}$ of heparin was administered to all animals immediately before the procedure. Animals in the treatment group received a $12-\mu \mathrm{g} / \mathrm{kg}$ dose of mouse recombinant erythropoietin (Sigma-Aldrich Co, St Louis, Mo) intraperitoneally 4 hours before operation. Sham ischemia mice had the aortic arch exposed through the same procedure, but without aortic crossclamping. Animals that did not receive erythropoietin pretreatment instead received placebo injections of normal saline solution. Animals were humanely killed at 50 hours after reperfusion, and their spinal cords were removed for analysis.

\section{Functional Locomotor Scores}

The Basso Mouse Scale for locomotion was used to quantify hind limb function in mice after ischemia, which ranges from a score of 0 for complete paraplegia to a score of 9 for normal function. ${ }^{10}$ Function was scored at $12,24,36$, and 48 hours after reperfusion.

\section{Histologic Analysis}

T-10 through L-3 spinal cord sections were harvested and preserved in $10 \%$ formalin for at least 24 hours before paraffin embedding, sectioning, and hemotoxylin and eosin staining. Sections were analyzed for the number of viable neurons.

\section{Western Blot Analysis}

Spinal cord specimens were preserved by flash freezing at $-80^{\circ} \mathrm{C}$. Specimens were then homogenized in ethylenediaminetetraacetic acid-free complete lysis-M buffer (Roche Diagnostics, Indianapolis, Ind), and protein quantification was performed with a Bradford Assay kit (Thermo Fisher Scientific Inc, Rockford, Ill.). Standard quantities of protein were loaded onto a $4 \%$ to $20 \%$ tris(hydroxymethyl)aminomethane (Tris) hydrochloride gradient gel (Bio-Rad Laboratories, Inc, Hercules, Calif), and membranes were run in Tris-glycine running buffer. Protein from gels was then transferred to a nitrocellulose membrane and blocked in 5\% nonfat milk for 1 hour. The membrane was then incubated in 1:500 mouse anti-human $\mathrm{HIF} 1 \alpha$ primary antibody overnight at $4{ }^{\circ} \mathrm{C}$. After primary incubation, membranes were washed 3 times for 5 minutes each in Trisbuffered saline solution. Membranes were then incubated at room temperature in 1:5000 horseradish peroxidase-linked secondary antibodies for 1 hour. Membranes were then rinsed with Tris-buffered saline solution, and target bands were developed with enhanced chemiluminescence and exposed on film. Densitometry was performed on all samples with ImageJ software (National Institutes of Health, Bethesda, Md).

\section{Statistics}

Statistics were performed with analysis of variance with a Fisher post hoc analysis for functional scores. Functional scores after ischemia-reperfusion were compared at all time points. Densitometry data was analyzed with the Student $t$ test.

\section{RESULTS}

Mice recovering from anesthesia had initial functional deficits, but mice in the treatment group $(n=7)$ regained significant function relative to those with no treatment $(n=7)$. During the next 48 hours, this improvement remained in the treatment group. Sham ischemia mice $(\mathrm{n}=6)$ had no decline in function at any time point studied (Figure 1). Overall, all mice without treatment had permanent paraplegia. In contrast, $43 \%$ of mice with erythropoeitin pretreatment regained full function. This effect was observed indefinitely in animals observed longer than 48 hours (data not presented).

Spinal cord sections showed decreased neuron viability after ischemia and reperfusion with no erythropoietin pretreatment (Figure 2). Qualitatively, sham ischemia mice

Functional Neurologic Outcomes After Aortic Cross Clamping

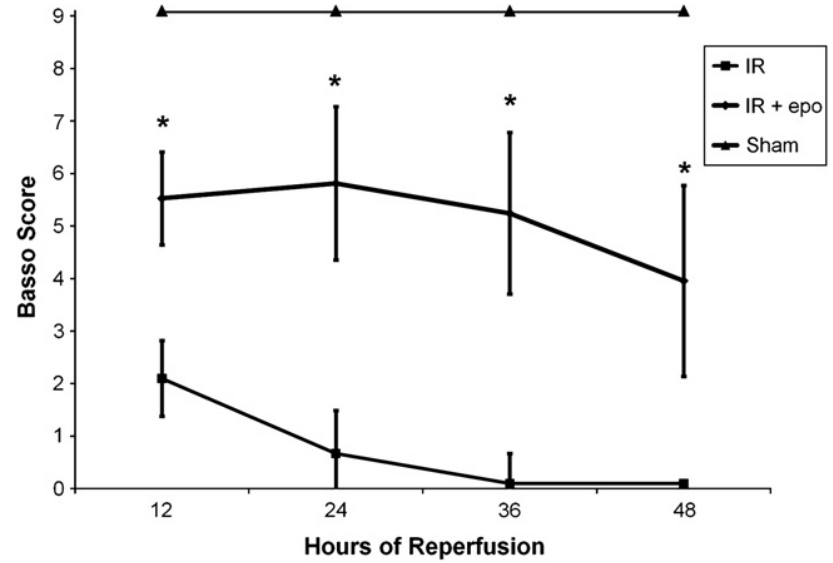

FIGURE 1. Hind limb motor function after spinal cord ischemia-reperfusion $(I R)$ or sham ischemia: 9 represents perfect function, whereas 0 represents no function. Mice receiving erythropoietin pretreatment (epo) showed significant improvement in function at all time points. Asterisk indicates $P<.05$ by analysis of variance. 

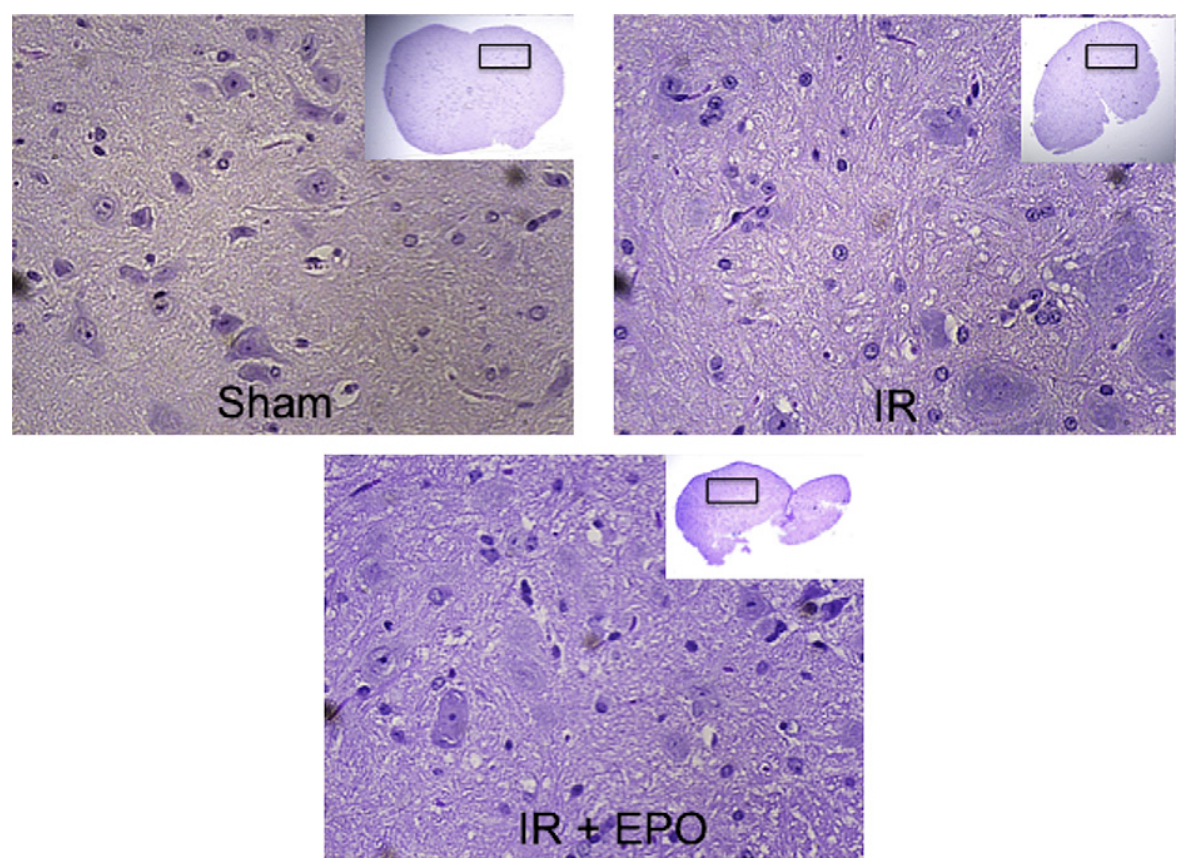

FIGURE 2. Hematoxylin and eosin staining of T9 to L3 spinal cord sections after 50 hours of reperfusion. Anterior horn cells are shown, with increased neuron viability in control (Sham) and erythropoietin-pretreated $(I R+E P O)$ groups relative to group with untreated ischemia-reperfusion $(I R)$.

and erythropoietin-pretreated mice showed increased neuron viability. Mice subjected to ischemia-reperfusion, including erythropoeitin-treated mice, had increased vacuolization relative to sham ischemia mice. Neurons in the erythropoietin-treated group, however, had decreased retraction of the nuclei and preservation of nuclear structures, including the nucleolus, indicating increased viability. In addition, inflammatory cells were increased in the ischemia-reperfusion group relative to both sham ischemia and erythropoietin-treated groups.

HIF1 $\alpha$ was detectable in normal (sham ischemia), treated, and untreated spinal cords. No difference in level of protein expression was detectable at 48 hours of reperfusion (Figure 3).

\section{DISCUSSION}

This study demonstrates the potential of erythropoietin as a pharmacologic adjunct to prevent spinal cord injury after aortic surgery. Erythropoietin has shown promise previously in brain protection after various types of insults. ${ }^{5,11,12}$ This has been validated not only through animal models but also in human trials. Beneficial effects are seen in diseases ranging from amyotrophic lateral sclerosis to subarachnoid hemorrhage. In the case of aneurysmal subarachnoid hemorrhage in human beings, erythropoietin treatment decreased delayed ischemic deficits after new ischemic events from $40.0 \%$ to $7.5 \% .{ }^{11}$ Although decreased vasospasm seems to be attributable to the protective effect in this study, the mechanisms by which this neuroprotection occurs in general is not known. Educated speculation attri- butes this effect to the broad categories of antiapoptotic, anti-inflammation, neurogenesis, and anti-oxidative stress mechanisms. ${ }^{13}$

Little is known about the mechanisms of neuroprotection of erythropoietin in the brain, and even less is known about the mechanisms by which neuroprotection occurs in the spinal cord. The same antiapoptotic, anti-inflammatory, neurogenic, and anti-oxidative stress mechanisms as in the brain have been proposed by several authors, but the evidence for these mechanisms is less substantial in the spinal cord. ${ }^{13}$ Rabbit and rat models of spinal cord ischemia-reperfusion have suggested a beneficial role of erythropoietin; again,

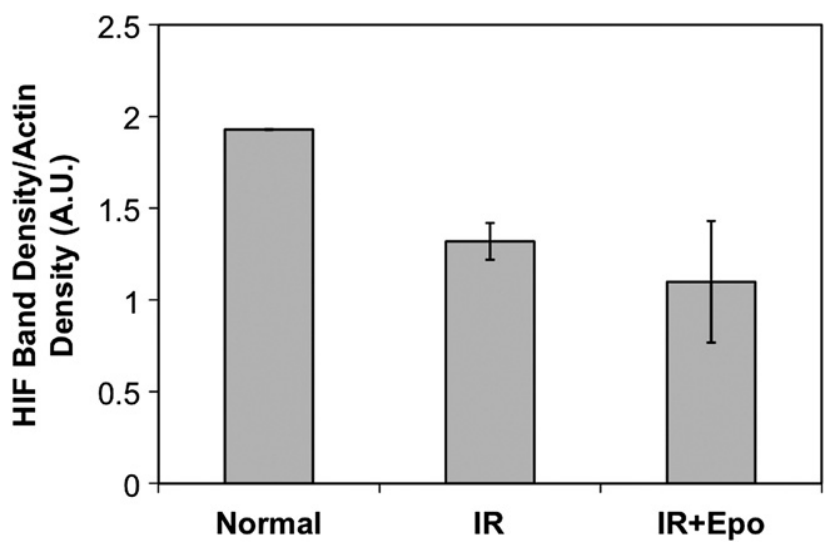

FIGURE 3. Densitometric analysis of hypoxia-inducible factor $1 \alpha(H I F)$ immunoblotting showing no significant difference in protein expression among sham ischemia (Normal), unmediated ischemia-reperfusion (IR), and erythropoietin-treated $(I R+E p o)$ groups. A.U., Arbitrary units. 
however, mechanisms remain largely unexplored. ${ }^{14,15}$ These models showed significant injury attenuation for dense, immediate paraplegia; however, the delayed, progressive injuries from this study were not addressed.

In addition to providing a model for the study of progressive neurologic injury, this murine model provides several advantages. Most importantly, this model allows for genetic manipulations of mice, which will facilitate further elucidation of the molecular mechanisms behind our findings. The short ischemic time required to produce an injury, attributable to the high metabolic rate in the mouse, allows faster experimentation at lower costs. In addition, the spinal cord vasculature in the mouse is more similar to that of a human than are other well-established animal models. ${ }^{8}$

These data demonstrate that erythropoietin pretreatment in a mouse model of spinal cord ischemia and reperfusion is beneficial in reducing both immediate and delayed neurologic injury. Unlike in previous reports, control mice in this series showed an initial injury that progressed to dense paraplegia by 48 hours. In contrast, erythropoietinpretreated mice showed improvement in both initial injury and delayed-onset paraparesis and paraplegia. All mice that underwent ischemia and reperfusion in our model had complete paraplegia develop without erythropoietin pretreatment. A significant number of mice with pretreatment recovered full neurologic function. These observations alone merit further investigation into the mechanisms and clinical applications of erythropoeitin treatment.

We were not able to observe a difference in HIF $1 \alpha$ protein expression at the 48 hour time point. This finding does not, however, exclude the possibility of this protein's involvement in neuroprotective mechanisms. Models of renal ischemia and reperfusion have shown increased expression of HIF $1 \alpha$ at 24 and 72 hours after ischemia-reperfusion in human cell lines. In light of the vast differences in metabolic rates between mice and human beings, however as well as inherent differences between in vitro and in vivo experiments, differential HIF1 $\alpha$ expressions may be observable at shorter intervals after reperfusion in our model. ${ }^{6}$

The immediate and delayed injuries seen after spinal cord ischemia-reperfusion can be explained by breaking down the mechanism into 2 components. The initial injury may be caused by a metabolic (or ischemic) insult to the spinal cord, whereas the progressive injury may be attributable to the metabolic insult combined with a secondary inflammatory response. Inferring mechanistic implications from our data on the basis of this 2-component model of injury is speculative but not unsupported by the literature. This injury in the brain has been hypothesized to be due to early injury secondary to resident inflammatory cells (mainly microglia), with a secondary response caused by recruited inflammatory mediators and proinflammatory cells. ${ }^{16}$ Of note, erythropoeitin derivatives have been known to protect tissue whether delivered before or after injury. ${ }^{17}$ These findings imply that erythropoeitin may act to attenuate both ischemic insult and reperfusion injury.

Erythropoietin pretreatment for patients undergoing thoracic aortic surgery is easily applied. The potential thrombosis issue may be negated by the requisite heparinization. Moreover, thrombosis occurs in patients with hematocrits greater than $40 \%$, which is clearly not an issue after these procedures. In fact, the hematopoietic effect of erythropoeitin may actually decrease the need for transfusion in the postoperative period. Many potential side effects of erythropoietin may actually prove beneficial in this setting.

Although our results are promising, they are not without limitations. A recent double-blind, placebo-controlled trial showed increased mortality and no advantageous effects in patients treated with erythropoietin in acute ischemic stroke. ${ }^{18}$ Although this finding does hamper enthusiasm for treating spinal cord ischemia-reperfusion injury with erythropoietin, the mechanisms of injury and patient conditions in these clinical scenarios are significantly different and provide no reason to believe that this same effect would become evident in aortic surgical patients, given the 20 year safety record of this drug. Our small sample size also limits the applicability of this study, although larger sample sizes can easily be produced to overcome this dilemma. The altitude at which our center is located may also make results difficult to reproduce at lower elevations; however, the overall mechanistic implications are still applicable.

In summary, this study provides evidence that erythropoietin may affect both the ischemic and reperfusion injuries related to thoracic aortic surgery. Further, study regarding the mechanisms of neuroprotection by erythropoietin may lead to direct benefit for patients while expanding our understanding of spinal cord ischemia-reperfusion injury.

\section{References}

1. Webb JD, Coleman ML, Pugh CW. Hypoxia, hypoxia-inducible factors (HIF), HIF hydroxylases and oxygen sensing. Cell Mol Life Sci. 2009;66:3539-54.

2. Joyeux-Faure M. Cellular protection by erythropoietin: new therapeutic implications? J Pharmacol Exp Ther. 2007;323:759-62.

3. Hasselblatt M, Ehrenreich H, Sirén AL. The brain erythropoietin system and it potential for therapeutic exploitation in brain disease. J Neurosurg Anesthesiol. 2006;18:132-8.

4. Minnerup J, Heidrich J, Rogalewski A, Schäbitz WR, Wellmann J. The efficacy of erythropoietin and its analogues in animal stroke models: a meta-analysis. Stroke. 2009;40:3113-20.

5. Wang Y, Zhang ZG, Rhodes K, Renzi M, Zhang RL, Kapke A, et al. Post-ischemic treatment with erythropoietin or carbamylated erythropoietin reduces infarction and improves neurological outcome in a rat model of focal cerebral ischemia. Br J Pharmacol. 2007;151:1377-84.

6. Imamura R, Moriyama T, Isaka Y, Namba Y, Ichimaru N, Takahara S, et al Erythropoietin protects the kidneys against ischemia reperfusion injury by activating hypoxia inducible factor- $1 \alpha$. Transplantation. 2007;83:1371-9.

7. Shi H. Hypoxia inducible factor 1 as a therapeutic target in ischemic stroke. Curr Med Chem. 2009;16:4593-600.

8. Lang-Lazdunski L, Matsushita K, Hirt L, Waeber C, Vonsattel JP, Moskowitz MA, et al. Spinal cord ischemia. Development of a model in the mouse. Stroke. 2000;31:208-13.

9. Stone DH, Conrad MF, Albadawi H, Entabi F, Stoner MC, Cambria RP, et al. Effect of PJ34 on spinal cord tissue viability and gene expression in a murine mode of thoracic aortic reperfusion injury. Vasc Endovascular Surg. 2009;43:444-51. 
10. Basso DM, Fisher LC, Anderson AJ, Jakeman LB, McTigue DM, Popovich PG. Basso Mouse Scale for locomotion detects differences in recovery after spinal cord injury in five common mouse strains. J Neurotrauma. 2006;23:635-59.

11. Tseng MY, Hutchinson PJ, Richards HK, Czosnyka M, Pickard JD, Erber WN, et al. Acute systemic erythropoietin therapy to reduce delayed ischemic deficits following aneurysmal subarachnoid hemorrhage: a Phase II randomized, doubleblind, placebo-controlled trial. Clinical article. J Neurosurg. 2009;111:171-80.

12. Talving P, Lustenberger T, Kobayashi L, Inaba K, Barmparas G, Schnüriger B, et al. Erythropoiesis stimulating agent administration improves survival after severe traumatic brain injury: a matched case control study. Ann Surg. 2010;251:1-4.

13. Byts N, Sirén AL. Erythropoietin: a multimodal neuroprotective agent. Exp Transl Stroke Med. 2009;21;1:4.

14. Sönmez A, Kabakçi B, Vardar E, Gürel D, Sönmez U, Orhan YT, et al. Erythropoietin attenuates neuronal injury and potentiates the expression of pCREB in anterior horn after transient spinal cord ischemia in rats. Surg Neurol. 2007;68: 297-303.

15. Celik M, Gökmen N, Erbayraktar S, Akhisaroglu M, Konakc S, Ulukus C, et al. Erythropoietin prevents motor neuron apoptosis and neurologic disability in experimental spinal cord ischemic injury. Proc Natl Acad Sci U S A. 2002;99: 2258-63.

16. Jin R, Yang G, Li G. Inflammatory mechanisms in ischemic stroke: role of inflammatory cells. J Leukoc Biol. 2010;87:779-89.

17. Leist M, Ghezzi P, Grasso G, Bianchi R, Villa P, Fratelli M. Derivatives of erythropoietin that are tissue protective but not erythropoietic. Science. 2004;305: 239-42.

18. Ehrenreich H, Weissenborn K, Prange H, Schneider D, Weimar C, Wartenberg K, et al. Recombinant human erythropoietin in the treatment of acute ischemic stroke. Stroke. 2009;40:e647-56.

\section{Discussion}

Dr John S. Ikonomidis (Charleston, SC). Drs Burdon and Fullerton, I have no relationships to disclose with regard to this discussion. I thank Ross Bremner and the Western Thoracic Surgical Association for the opportunity to discuss this presentation today, and I rise to congratulate Drs Smith and Reece and the University of Colorado group for a really compelling characterization of this novel and interesting spinal cord protection phenomenon that we are really just starting to understand.

Dr Smith, I have a couple of comments and questions. I would like you to answer each question as I give it.

First, at first glance at this data, I am astounded that 5 minutes of normothermic ischemia caused uniform paralysis in every untreated animal studied. This is a devastating amount of damage that certainly would not be expected to occur in a similar clinical scenario in human beings, so I wonder whether you could comment on any potential anatomic or vascular-related differences between the murine model and human anatomy that could impede the extrapolation of these results to human patients into the clinical setting.

Dr Smith. Absolutely. Thank you for your kind comments. We feel that the most important thing is that the functional injury that we observe after aortic occlusion is very similar to what is seen clinically in human patients. The spinal cord anatomy and vasculature of a mouse is actually very similar to those of human beings, more than other animal models that have been used. Finally, the metabolic rate of a mouse is so much faster than that of a human being that you might actually expect that very short ischemic times such as those we used to have a much more pronounced effect.

Dr Ikonomidis. Thank you. Some logistic issues related to erythropoietin. How did you arrive at the timing and dosage of erythropoietin used in this study?

Dr Smith. We looked at the other literature, on the brain, and there have been many studies of the brain that outline different tim- ing of administration and dosing, and we chose from there. In addition, we do not know the optimal timing or dosage, and we are doing further studies now to try to figure that out.

Dr Ikonomidis. How much does this cost in a mouse, and if you were to extrapolate that dose to human beings, how much would it cost if we were to use this clinically?

Dr Smith. I have not broken down the cost for a mouse. If you extrapolate the dose we used to a human dose, it would be about $120 \mathrm{unit} / \mathrm{kg}$, so that would be about $\$ 200$ to $\$ 300$ per dose.

Dr Ikonomidis. It occurs to me that a single dose of erythropoietin, given the timing of this study, probably would not cause significant hematopoietic effects and is probably pretty benign. So in light of what we know already about it, should we start doing this in every case every time we do a descending thoracic replacement or thoracoabdominal? What is the harm in not giving it?

Dr Smith. I think that we need to further elucidate the mechanisms before we move into human trials, but because of the safety profile of erythropoietin and in the general population much less the aortic surgical population, I think it is very promising for treating every patient in the future.

Dr Ikonomidis. Your answer to that question is a good segue to the next one. There is relatively little literature on this subject, but the literature that is available shows this effect quite uniformly, so I think that it is reasonable to say that it really exists, and I think it is time to move forward and really start hammering out mechanisms. Your HIF $1 \alpha$ data were not particularly compelling, but there are a variety of other potential mechanisms. These include anti-inflammatory effects, anti-apoptotic effects, modulation of oxidative stress, neurohormonal modulation of vascular tone, and even increased phosphorylation of certain upstream protective transcription factors, such as CREB. Are you leaning toward any of these particular mechanisms, or do you have a different mechanism in mind? How do you plan to study that?

Dr Smith. As you mentioned, there are several possible mechanisms that may be responsible for this effect. Although we did not observe a change in HIF1 $\alpha$ at 48 hours, we still believe that it may be responsible, so we would like to look at and are looking at different time points to see whether we can show different expression after erythropoietin administration.

With respect to other mechanisms that may be involved, we are also looking at cytokine expression as a function of time after ischemia and reperfusion to see whether we can determine the important proinflammatory and anti-inflammatory cytokines that are responsible for the injury. Also, as I mentioned briefly, there are erythropoietin derivatives that do not have any hematopoietic effect but do have a cytoprotective effect, so we are using these to try to tease out the actual neuroprotective effects of these drugs.

Dr Jeffrey Gaca (Durham, NC). We often do these operations under hypothermia. Do you think that maybe you could reproduce this experiment under hypothermia and get different results, maybe even better results? A lot of times we don't do them under normothermia now.

Dr Smith. Absolutely, that is a great question. I think that if we did these experiments under hypothermic conditions, we might see even more neuroprotection. We do not know that for sure, because we have only done this under normothermia, but that would be a reasonable thing to try because that is what is done clinically. 